\title{
ON THE EFFECTIVE BLOCK SIZE IN HARPER'S THEOREM
}

\author{
William M. Y. Goh and Jet Wimp
}

\begin{abstract}
ABSTR.ACT. Let $s$ be a random set partition of $[n]$ and $X_{n}(\sigma)$ be the random variable marking the total number of blocks in $\sigma$. By employing a uniform probability on the sample space of random set partitions of $[n], L$. Harper proved a central limit theorem for $X_{n}(\sigma)$.

We determine the effective size of the block size in Harper's theorem, that is, the minimal block size for which the conclusion of Harper's theorem is still maintained. This size is expressed as a quotient of the roots of two transcendental equations.
\end{abstract}

\section{Introduction}

The notion of effective size appears naturally in many problems in combinatorial enumeration. To illustrate the point we take the following example. Let $\sigma$ be a random set partition of $[n]$, where $[n]=\{1,2, \ldots, n\}$, the set of first $n$ natural numbers. Let $X_{n}(\sigma)$ be the total number of blocks in $\sigma$. Also let $u_{n}$ be the unique positive root of $z e^{z}=n$. Define the random variable

$$
Y_{n}(\sigma):=\frac{X_{n}(\sigma)-M_{n}}{D_{n}}, \text { where } M_{n}=\frac{n}{u_{n}} \text { and } D_{n}=\sqrt{n / u_{n}^{2}} .
$$

We assume a uniform probability on the sample space of random set partitions of $[n]$. The well-known theorem of Harper [4] states that

$$
\operatorname{Prob}\left(Y_{n} \leq x\right) \rightarrow \frac{1}{\sqrt{2 \pi}} \int_{-\infty}^{x} e^{-t^{2} / 2} d t \quad \text { as } n \rightarrow \infty
$$

In other words, we have a version of a central limit theorem. The "effective size" of the blocks is the optimal size $k=k(n)$, so that if we count only those blocks in a random set partition $\sigma$ of sizes up to $k+L_{n}$ where $L_{n}$ is an arbitrary function that goes to infinity as $n \rightarrow \infty$, call this count $X_{n}^{(k)}(\sigma)$, then we still obtain the conclusion of Harper's theorem,

$$
\operatorname{Prob}\left(\frac{X_{n}^{(k)}-M_{n}}{D_{n}} \leq x\right) \rightarrow \frac{1}{\sqrt{2 \pi}} \int_{-\infty}^{x} e^{-t^{2} / 2} d t .
$$

To maintain asymptotic normality, it is not necessary to count the total number of blocks in a random set partition. We just have to count the number of blocks in $\sigma$ of sizes up to $k+L_{n}$. We emphasize that in (1.2) and (1.3) the mean $M_{n}$ and deviation

Received March 29, 1993, revised June 15, 1993.

1991 Mathematics Subject Classification: $41 \mathrm{~A} 60$.

Key words and phrases: set partitions, asymptotics, central limit theorem, block size, Harper's Theorem.

The first author was supported by the National Science Foundation under Grant \# DMS 9101753. 
$D_{n}$ are the same. Obviously $k$ is not uniquely determined. Different $k$ 's may differ by a constant.

One can define the concept of effective size for other combinatorial configurations, for instance, the effective size of blocks in random ordered set partitions [2] or the effective size of primes in the Kac-Erdös theorem [5] on the distribution of $\omega(n)$. Both are interesting problems to study. This paper deals with the effective block size in a random set partition of $[n]$. Our major theorem is the following:

Theorem 1. The effective size $k$ of blocks in a random set partition of $[n]$ is $k=$ $k(n)=u_{n} / r$ where $r$ is the unique positive root of $z / 2-\ln z-1=0$ in the unit interval $(0,1)$.

A rough estimate for $r$ is $2.4>1 / r>2$. This means $1 / r<e$. It is well-known that the mean of maximum block size of a random partition is $\sim e \ln n$, see [7]. Hence the effective size is smaller than the average maximum block size but the two are of the same asymptotic order. It is also well-known [6] that $u_{n}=\ln n-\ln \ln n+$ $o(1)$. Therefore, the second-order term $(-1 / r) \ln \ln n$ of the effective size cannot be dropped.

\section{An analytical formulation of the problem}

Our strategy for tackling the problem is straightforward. From the block index of a random set partition we have the following multivariate generating function (see Chapter 3 of [11]):

$$
\sum_{n \geq 0} \frac{B(n)}{n !} E\left(t_{1}^{X_{n 1}} t_{2}^{X_{n 2}} \cdots t_{n}^{X_{n n}}\right) x^{n}=\exp \left(\sum_{m \geq 1} \frac{t_{m} x^{m}}{m !}\right),
$$

where $X_{n i}(\sigma)$ is the number of blocks of size $i$ in $\sigma, 1 \leq i \leq n$. Let $E(X)$ denote the usual expectation of the random variable $X$, and $B(n)$ the $n$-th Bell number, i.e., the total number of set partitions of $[n]$. In (2.1), set $t_{i}=t$ for $1 \leq i \leq k$ and $t_{i}=1$ for $i \geq k+1$. Thus

$$
\sum_{n \geq 0} \frac{B(n)}{n !} E\left(t^{\bar{X}_{n, k}}\right) x^{n}=\exp \left(t \sum_{1 \leq m \leq k} \frac{x^{m}}{m !}\right) \exp \left(\sum_{m \geq k+1} \frac{x^{m}}{m !}\right),
$$

where the random variable $\bar{X}_{n, k}:=\sum_{j=1}^{k} X_{n, j}$ marks the total number of blocks of sizes up to $k$.

Introducing $S_{n}(x):=\sum_{j=0}^{n}\left(x^{j} / j !\right)$, the $n$-th partial sum of $e^{x}$, and $R_{n+1}(x):=$ $e^{x}-S_{n}(x)$, the remainder of $e^{x}$ starting with the term $x^{n+1}$, we have

$$
\sum_{n \geq 0} \frac{B(n)}{n !} E\left(t^{\bar{X}_{n \cdot k}}\right) x^{n}=e^{t\left(S_{k}(x)-1\right)} e^{R_{k+1}(x)} .
$$

By Cauchy's integral formula

$$
E\left(t^{\bar{X}_{n, k}}\right)=\frac{n !}{B(n)} \frac{1}{2 \pi i} \oint_{C} \frac{\exp \left(t\left(S_{k}(x)-1\right)+R_{k+1}(x)\right)}{x^{n+1}} d x
$$

where $C$ is any simple closed contour encircling the origin. 
Now set $t=e^{\xi}$ in $(2.3), \xi$ real,

$$
E\left(e^{\xi \bar{X}_{n \cdot k}}\right)=\frac{n !}{B(n)} \frac{1}{2 \pi i} \oint_{C} \frac{\exp \left(e^{\xi}\left(S_{k}(x)-1\right)+R_{k+1}(x)\right)}{x^{n+1}} d x .
$$

We normalize $\bar{X}_{n, k}$ the same way as in Harper's theorem. Thus

$$
\begin{aligned}
& E\left(\exp \left(\xi \frac{\bar{X}_{n, k}-M_{n}}{D_{n}}\right)\right) \\
& \quad=e^{-\xi M_{n} / D_{n}} \frac{n !}{B(n)} \frac{1}{2 \pi i} \oint_{C} \frac{\exp \left(e^{\xi / D_{n}}\left(S_{k}(x)-1\right)+R_{k+1}(x)\right)}{x^{n+1}} d x .
\end{aligned}
$$

Our goal is clear. We must find an optimal $k=k(n)$ so that the right-hand side of (2.4) approaches $e^{\xi^{2} / 2}$ as $n \rightarrow \infty$. By the continuity theorem in probability [3] we then will have proved the theorem. The technique that is required to finish the proof is very involved. Basically, we apply the saddle point method to approximate the integral in (2.4). Unfortunately, the integrand is a complicated function of $n$ and $x$. In such problems, uniformity of the approximation is always a major issue that must be confronted in the analysis.

\section{Lemmas of approximation}

This section collects some useful approximations that are relevant to the problem. Throughout this paper $\xi$ is always held fixed. To simplify the matter, we need to define some notation. Let

$$
I_{n, k}=\frac{1}{2 \pi i} \oint_{C} \exp \left(g_{n, k}(x)\right) \frac{d x}{x}
$$

where

$$
\begin{aligned}
g_{n, k}(x) & =e^{\xi / D_{n}}\left(S_{k}(x)-1\right)+R_{k+1}(x)-n \ln x \\
& =e^{x}-n \ln x+\left(e^{\xi / D_{n}}-1\right) S_{k}(x)-e^{\xi / D_{n}} .
\end{aligned}
$$

A formal application of the saddle point method yields

$$
g_{n, k}^{\prime}(x)=e^{x}-\frac{n}{x}+\left(e^{\xi / D_{n}}-1\right) S_{k-1}(x) .
$$

Proposition 1. $g_{n, k}^{\prime}(x)$ as a function of $x$ defined on $(0, \infty)$ has a unique positive root $\rho_{n, k}$.

Proof. Define

$$
h_{n, k}(x)=x\left(e^{x}+\left(e^{\xi / D_{n}}-1\right) S_{k-1}(x)\right)
$$

on $(0, \infty)$. Differentiating with respect to $x$ yields

$$
h_{n, k}^{\prime}(x)=\left(R_{k}(x)+e^{\xi / D_{n}} S_{k-1}(x)\right)+x\left(R_{k-1}(x)+e^{\xi / D_{n}} S_{k-2}(x)\right) .
$$

Since each term in the above is positive, $h_{n, k}^{\prime}(x)>0$ on $(0, \infty)$. Notice that $h_{n, k}(0)=0$ and $h_{n, k}(\infty)=\infty$. This $h_{n, k}(x)$ is a continuous, strictly increasing function that maps $[0, \infty)$ to $[0, \infty)$. Hence, $h_{n, k}(x)=n$ has a unique positive root. 
Recall that $u_{n}$ is the unique positive root of $z e^{z}=n$. The following proposition describes the asymptotic behavior of $\rho_{n, k}$.

Proposition 2.

$$
\rho_{n, k}-u_{n}=-\frac{\xi}{D_{n}} \frac{S_{k-1}\left(\rho_{n, k}\right)}{e^{\rho_{n, k}}}+\frac{\xi}{D_{n} u_{n}} \frac{S_{k-1}\left(\rho_{n, k}\right)}{e^{\rho_{n, k}}}+O\left(\frac{1}{D_{n} \ln ^{2} n}\right),
$$

uniformly for all positive integers $k$.

Proof. We first show that $\rho_{n, k}-u_{n}=o(1)$ as $n \rightarrow \infty$, uniformly for all $k \geq 1$.

(1) First we assume $\xi \geq 0$. Returning to (3.2), one can easily show that

$$
x e^{x} \leq h_{n, k}(x) \leq h_{n, \infty}(x)=e^{\xi / D_{n}} x e^{x} .
$$

Hence $u_{n} \geq \rho_{n, k} \geq \nu_{n}$ where $\nu_{n}$ is the unique positive root of

$$
e^{\xi / D_{n}} x e^{x}=n .
$$

Thus

$$
\left|\rho_{n, k}-u_{n}\right| \leq\left|\nu_{n}-u_{n}\right|
$$

Observe that $\nu_{n}$ satisfies $\nu_{n} e^{\nu_{n}}=n e^{-\xi / D_{n}}$. Let $u_{t}$ be the unique positive root of $z e^{z}=t, t>0$. Then in terms of $u_{t}$ we have

$$
\nu_{n}=u_{n e^{-\xi / D_{n}}} .
$$

Using the formula $u_{t}=\ln t-\ln \ln t+o(1)$, we get

$$
\begin{aligned}
\nu_{n} & =\ln \left(n e^{-\xi / D_{n}}\right)-\ln \ln \left(n e^{-\xi / D_{n}}\right)+o(1) \\
& =\ln n-\ln \ln n+o(1) .
\end{aligned}
$$

This implies that

$$
\nu_{n}-u_{n}=o(1)
$$

Combining (3.9) with (3.6), we have

$$
\rho_{n, k}-u_{n}=o(1), \quad \text { uniformly in } k .
$$

(2) The case when $\xi<0$ can be dealt with similarly.

Now we perform a "bootstrap" computation. Since $\rho_{n, k}$ is a root of $h_{n, k}(x)=n$, we have

$$
n=\rho_{n, k}\left(e^{\rho_{n . k}}+\left(e^{\xi / D_{n}}-1\right) S_{k-1}\left(\rho_{n, k}\right)\right) .
$$

Also

$$
n=u_{n} e^{u_{n}}
$$

Dividing (3.11) by (3.12) gives

$$
1=\frac{\rho_{n, k}}{u_{n}} e^{-u_{n}}\left(e^{\rho_{n . k}}+\left(e^{\xi / D_{n}}-1\right) S_{k-1}\left(\rho_{n, k}\right)\right) .
$$

Taking logarithms, we arrive at

$$
0=\ln \frac{\rho_{n, k}}{u_{n}}-u_{n}+\rho_{n, k}+\ln \left(1+\left(e^{\xi / D_{n}}-1\right) S_{k-1}\left(\rho_{n, k}\right) e^{-\rho_{n, k}}\right) .
$$


Note that

$$
\begin{gathered}
\ln \frac{\rho_{n, k}}{u_{n}}=\ln \left(1+\frac{\rho_{n, k}-u_{n}}{u_{n}}\right)=\frac{\rho_{n, k}-u_{n}}{u_{n}}+O\left(\left|\frac{\rho_{n, k}-u_{n}}{u_{n}}\right|^{2}\right), \\
\text { and } 0<\frac{S_{k-1}\left(\rho_{n, k}\right)}{e^{\rho_{n, k}}}<1 .
\end{gathered}
$$

Thus (3.13) simplifies to

$$
\begin{array}{r}
0=\frac{\rho_{n, k}-u_{n}}{u_{n}}+O\left(\left|\frac{\rho_{n, k}-u_{n}}{u_{n}}\right|^{2}\right)+\left(\rho_{n, k}-u_{n}\right) \\
+\left(e^{\xi / D_{n}}-1\right) \frac{S_{k-1}\left(\rho_{n, k}\right)}{e^{\rho_{n, k}}}+O\left(\left|e^{\xi / D_{n}}-1\right|^{2}\right)
\end{array}
$$

or

$$
0=\left(\rho_{n, k}-u_{n}\right)\left(1+\frac{1}{u_{n}}+O\left(\frac{1}{u_{n}^{2}}\right)\right)+\left(\frac{\xi}{D_{n}} \frac{S_{k-1}\left(\rho_{n, k}\right)}{e^{\rho_{n, k}}}+O\left(\frac{1}{D_{n}^{2}}\right)\right)
$$

uniformly for $k \geq 1$.

Solving (3.14) for $\rho_{n, k}-u_{n}$ gives

$$
\begin{aligned}
\rho_{n, k}-u_{n} & =\left(\frac{-\xi}{D_{n}} \frac{S_{k-1}\left(\rho_{n, k}\right)}{e^{\rho_{n, k}}}+O\left(\frac{1}{D_{n}^{2}}\right)\right)\left(1+O\left(\frac{1}{u_{n}}\right)\right) \\
& =\frac{-\xi}{D_{n}} \frac{S_{k-1}\left(\rho_{n, k}\right)}{e^{\rho_{n, k}}}+O\left(\frac{1}{D_{n} \ln n}\right) .
\end{aligned}
$$

We can now write $\rho_{n, k}-u_{n}=\left(-\xi / D_{n}\right)\left(S_{k-1}\left(\rho_{n, k}\right) / e^{\rho_{n, k}}\right)+\varepsilon_{n}$ with $\varepsilon_{n}=O\left(1 / D_{n} \ln n\right)$. We plug this into (3.13) and bootstrap again. Finally, we get the desired result,

$$
\varepsilon_{n}=\frac{\xi}{D_{n} u_{n}} \frac{S_{k-1}\left(\rho_{n, k}\right)}{e^{\rho_{n . k}}}+O\left(\frac{1}{D_{n} \ln ^{2} n}\right)
$$

Proposition 3. (A)

$$
\begin{gathered}
\frac{S_{k}(k x)}{e^{k x}}=\delta+\sqrt{\frac{2}{\pi}} \frac{\zeta x}{x-1} \operatorname{Erfc}(\sqrt{k} \zeta)\left(1+O\left(\frac{1}{\sqrt{k}}\right)\right), \text { where } \\
\delta:=\left\{\begin{array}{ll}
1, & 0 \leq x<1 \\
0, & x \geq 1
\end{array}, \quad \text { and } \zeta=|x-1-\ln x|^{1 / 2},\right.
\end{gathered}
$$

uniformly for $x \geq 0$ and

$$
\operatorname{Erfc}(\sigma):=\int_{\sigma}^{\infty} e^{-t^{2}} d t, \quad \sigma>0
$$

(B) Let $z$ be in a compact set $K$ of the open unit disk $\{z \in \mathbb{C}:|z|<1\}$. Then we have

$$
\frac{S_{k}(k z)}{e^{k z}}=1-\frac{1}{\sqrt{2 k \pi}} \frac{z}{1-z}\left(z e^{1-z}\right)^{k}\left(1+O\left(\frac{1}{k}\right)\right)
$$

uniformly for all $z \in K$. 
Proof. First we give the proof of (A). Recall that

$$
S_{n}(x):=\sum_{k=0}^{n} \frac{x^{k}}{k !}
$$

We can write

$$
\begin{aligned}
S_{n}(x) & =\frac{x^{n+1}}{n !} \int_{0}^{\infty} e^{-x t}(1+t)^{n} d t \\
& =\frac{e^{x}}{n !} \int_{x}^{\infty} e^{-t} t^{n} d t
\end{aligned}
$$

An integration by parts gives

$$
S_{n}(x)=\frac{x^{n}}{n !}+e^{x} Q(n, x),
$$

and

$$
S_{n}(n x)=\frac{(n x)^{n}}{n !}+e^{n x} Q(n, n x),
$$

in the notation of Temme [9]. The results in that reference give the asymptotic expansion,

$$
S_{n}(n x)=\frac{(n x)^{n}}{n !}+e^{n x}\left\{\frac{1}{\sqrt{\pi}} \operatorname{Erfc}[\varepsilon \sqrt{n} \zeta]+\frac{\left(e^{-x} x e\right)^{n}}{\sqrt{2 \pi n}}\left[c_{0}+\frac{c_{1}}{n}+\frac{c_{2}}{n^{2}}+\cdots\right]\right\}
$$

where

$$
\zeta:=|x-1-\ln x|^{1 / 2}, \quad \varepsilon:=\operatorname{sgn}(x-1), \quad c_{0}=\frac{1}{x-1}-\frac{\varepsilon}{\sqrt{2} \zeta} .
$$

This asymptotic expansion is uniform for $x \geq 0$. Although there may appear to be a problem near $x=1$, there isn't, since,

$$
c_{0}=\frac{1}{3}+O[(x-1)]
$$

Using Stirling's formula on the first term gives

$$
\frac{S_{n}(n x)}{e^{n x}}=\frac{1}{\sqrt{\pi}} \operatorname{Erfc}[\varepsilon \sqrt{n} \zeta]+\frac{e^{-n \zeta^{2}}}{\sqrt{2 \pi n}}\left(\frac{x}{x-1}-\frac{\varepsilon}{\sqrt{2} \zeta}\right)\left(1+O\left(\frac{1}{n}\right)\right),
$$

uniformly for $x \geq 0$. Now consider Gautschi's inequality [1, see 7.1.13],

$$
\frac{1}{y+\sqrt{y^{2}+2}}<e^{y^{2}} \operatorname{Erfc} y \leq \frac{1}{y+\sqrt{y^{2}+4 / \pi}}, \quad y \geq 0 .
$$

The usefulness of this inequality lies in its uniformity. We may write

$$
e^{y^{2}} \operatorname{Erfc} y=\frac{1}{y+\sqrt{y^{2}+a(y)}}, \quad \frac{4}{\pi} \leq a(y)<2, y \geq 0 .
$$

For our purposes we rewrite the above result as

$$
e^{-y^{2}}=(2 y+b(y)) \operatorname{Erfc} y, \quad b(y)=\frac{a(y)}{y+\sqrt{y^{2}+a(y)}}, \quad y \geq 0 .
$$

We see that

$$
0<b(y) \leq \sqrt{2}
$$


The second term may be written

$$
\begin{aligned}
& \frac{e^{-n \zeta^{2}}}{\sqrt{2 \pi n}}\left(\frac{x}{x-1}-\frac{\varepsilon}{\sqrt{2} \zeta}\right)\left(1+O\left(\frac{1}{n}\right)\right) \\
& \quad=\frac{1}{\sqrt{2 \pi n}} \operatorname{Erfc}[\sqrt{n} \zeta]\left(\frac{x}{x-1}-\frac{\varepsilon}{\sqrt{2} \zeta}\right)(2 \sqrt{n} \zeta+b(\sqrt{n} \zeta))\left(1+O\left(\frac{1}{n}\right)\right) \\
& \quad=\frac{1}{\sqrt{2 \pi n}} \operatorname{Erfc}[\sqrt{n} \zeta]\left(\frac{x}{x-1}-\frac{\varepsilon}{\sqrt{2} \zeta}\right)\left(\sqrt{\frac{2}{\pi}} \zeta+\frac{b(\sqrt{n} \zeta)}{\sqrt{2 \pi n}}+O\left(\frac{1}{n}\right)\right) \\
& \quad=\operatorname{Erfc}[\sqrt{n} \zeta]\left\{\sqrt{\frac{2}{\pi}} \frac{\zeta x}{x-1}-\frac{\varepsilon}{\sqrt{\pi}}+O\left(\frac{1}{\sqrt{n}}\right)\right\} .
\end{aligned}
$$

There are two cases to consider, $\varepsilon=1$ and $\varepsilon=-1$. For the latter, we use the fact that

$$
\operatorname{Erfc} y+\operatorname{Erfc}(-y)=\sqrt{\pi}
$$

Accounting for each case and putting (3.19) in (3.18) gives the final result,

$$
\frac{S_{n}(n x)}{e^{n x}}=\delta+\sqrt{\frac{2}{\pi}} \frac{\zeta x}{x-1} \operatorname{Erfc}(\sqrt{n} \zeta)\left(1+O\left(\frac{1}{\sqrt{n}}\right)\right),
$$

uniformly for $x \geq 0$, where

$$
\delta:= \begin{cases}1, & 0 \leq x<1 \\ 0, & x \geq 1\end{cases}
$$

Note that since

$$
\zeta=\frac{|x-1|}{\sqrt{2}}(1+O(x-1))
$$

the lead term above is continuous at $x=1$, and yields, in fact, $1 / 2$.

(B) follows from (A) when $z \geq 0$. The proof for a complex $z$ can be found in [8], [10].

The following proposition is needed in Section 4.

\section{Proposition 4.}

$$
\frac{S_{k}(k x)}{e^{k x}} \leq \frac{1}{2}+O\left(\frac{1}{\sqrt{k}}\right)
$$

uniformly for $x \geq 1$.

Proof. Let $\zeta=|x-1-\ln x|^{1 / 2}, F_{k}(x):=x \operatorname{Erfc}(\sqrt{k} \zeta)$, and $H(x):=\sqrt{2 / \pi} \zeta /(x-1)$. By Proposition 3, we have

$$
\frac{S_{k}(k x)}{e^{k x}}=H(x) F_{k}(x)\left(1+O\left(\frac{1}{\sqrt{k}}\right)\right) .
$$

It is easy to prove that $H(x)$ is a decreasing function on $[1, \infty)$. Our immediate goal is to prove that there exists a $k_{0}$ such that for all $k \geq k_{0}, F_{k}(x)$ is a decreasing function of $x$ on $[1, \infty)$.

Now

$$
F_{k}^{\prime}(x)=\operatorname{Erfc}(\sqrt{k} \zeta)-\frac{\sqrt{k}}{2 H(x)} e^{-k \zeta^{2}}
$$


Note that

$$
\begin{aligned}
\operatorname{Erfc}(\sigma): & =\int_{\sigma}^{\infty} e^{-t^{2}} d t \leq e^{-\sigma^{2}}+e^{-(\sigma+1)^{2}}+e^{-(\sigma+2)^{2}}+\cdots \\
& =e^{-\sigma^{2}} \sum_{j=0}^{\infty} e^{-2 \sigma j-j^{2}} \leq e^{-\sigma^{2}} \sum_{j=0}^{\infty} e^{-j^{2}}
\end{aligned}
$$

Since $H(x)$ is decreasing on $[1, \infty)$, using (3.21) and (3.22), we have

$$
F_{k}^{\prime}(x) \leq e^{-k \zeta^{2}} \sum_{j=0}^{\infty} e^{-j^{2}}-\frac{\sqrt{k}}{2 H(1)} e^{-k \zeta^{2}}<0 .
$$

Thus there exists a $k_{0}$ such that for all $k \geq k_{0} F_{k}(x)$ is strictly decreasing on $[1, \infty)$. This implies $H(x) F_{k}(x)$ is decreasing on $[1, \infty)$. Returning to $(3.20)$, we see

$$
\frac{S_{k}(k x)}{e^{k x}} \leq H(1) F_{k}(1)\left(1+O\left(\frac{1}{\sqrt{k}}\right)\right) .
$$

The proposition follows from observing that $H(1) F_{k}(1)=1 / 2$.

Recall the random variable $\bar{X}_{n, k}:=\sum_{j=1}^{k} X_{n, j}$ which marks the total number of blocks of sizes up to $k$.

Proposition 5. For the value of the expectation, we have

$$
E\left(\bar{X}_{n, k}\right)=S_{k}\left(u_{n}\right)+O\left(\frac{S_{k}\left(u_{n}\right)}{u_{n}}\right)
$$

uniformly for $k \leq \sqrt{2} u_{n}$.

Proof. Differentiating (2.3) with respect to $t$ followed by setting $t=1$, we have

$$
E\left(\bar{X}_{n, k}\right)=\frac{n !}{e B(n)} \frac{1}{2 \pi i} \oint_{C} \exp \left(e^{x}-n \ln x\right)\left(S_{k}(x)-1\right) \frac{d x}{x} .
$$

From this point on we follow deBruijn closely (see [6]). We shall use Szegö's approximation to tame the behavior of $S_{k}(x)$. Let $h(x)=e^{x}-n \ln x$. The saddle points are roots of $h^{\prime}(x)=0$, i.e., roots of $x e^{x}=n$. Thus $u_{n}$ is a saddle point. The difficulty in proving the statement arises from non-uniformity and the unboundedness of $S_{k}\left(u_{n}\right)$. In the sequel, we shall show the contribution from all other saddle points is negligible. We may replace the integration contour $C$ by a segment of the vertical line through $u_{n}$, and complete it to a closed contour by adding a large semi-circle. And if we make the radius $R$ of the semi-circle tend to infinity, its contribution to the integral (3.25) tends to zero, the factor $S_{k}(x) / x^{n+1}$ being $O\left(R^{k-n-1}\right)$ whereas exp $e^{x}$ is bounded in the half-plane $\operatorname{Re} x \leq u_{n}$. Therefore, the integral in (3.25) may be replaced by $\int_{u_{n}-i \infty}^{u_{n}+i \infty}$. Writing $x=u_{n}+i y$, we obtain

$$
E\left(\bar{X}_{n, k}\right)=\frac{n !}{2 \pi e B(n)} \exp \left(e^{u_{n}-n \ln u_{n}}\right) \int_{-\infty}^{\infty} \exp (\psi(y))\left(\frac{S_{k}\left(u_{n}+i y\right)-1}{u_{n}+i y}\right) d y
$$

where

$$
\psi(y)=e^{u_{n}}\left(\left(e^{i y}-1\right)-u_{n} \ln \left(1+i y u_{n}^{-1}\right)\right)
$$


Notice that $|\exp \psi(y)|=\exp (\operatorname{Re} \psi(y))$. We have to study

$$
\operatorname{Re} \psi(y)=e^{u_{n}}\left(-1+\cos y-u_{n} \ln \left(1+y^{2} u_{n}^{-2}\right)^{1 / 2}\right) .
$$

We now show that in $(3.26)$ we can restrict ourselves essentially to the interval $[-\pi, \pi]$. First, we estimate the contribution for $y \geq \pi$. The situation when $y \leq-\pi$ can be dealt with similarly.

If $\pi<y<u_{n}$, then we have $\ln \left(1+y^{2} / u_{n}^{2}\right)>\frac{1}{2} y^{2} / u_{n}^{2}$ and $\left|S_{k}\left(u_{n}+i y\right)-1\right|<$ $2 S_{k}\left(\sqrt{2} u_{n}\right)$ and therefore

$$
\left|\int_{\pi}^{u_{n}} \exp \psi(y) \frac{S_{k}\left(u_{n}+i y\right)-1}{u_{n}+i y} d y\right|<\frac{2 u_{n}}{\left|u_{n}+i \pi\right|} \exp \left(-\pi^{2} e^{u_{n}} /\left(4 u_{n}\right)\right) S_{k}\left(\sqrt{2} u_{n}\right) .
$$

Note that $S_{k}(x)<e^{x}$ for all $x>0$ and all $k$. Thus

$$
\begin{aligned}
\left|\int_{\pi}^{u_{n}} \exp \psi(y) \frac{S_{k}\left(u_{n}+i y\right)-1}{u_{n}+i y} d y\right| & =O\left(\exp \left(-\pi^{2} e^{u_{n}} /\left(4 u_{n}\right)+\sqrt{2} u_{n}\right)\right) \\
& =O\left(\exp \left(-e^{u_{n}} / u_{n}\right)\right)
\end{aligned}
$$

If $y>u_{n}$, we use $1+y^{2} / u_{n}^{2}>2 y / u_{n}$. Putting $y=u_{n} x$, we get

$$
\left|\int_{u_{n}}^{\infty} \exp \psi(y) \frac{S_{k}\left(u_{n}+i y\right)-1}{u_{n}+i y} d y\right| \leq 2 \int_{1}^{\infty} \exp \left(\frac{-e^{u_{n}} u_{n} \ln (2 x)}{2}\right) \frac{\left|S_{k}\left(u_{n}(1+i x)\right)\right|}{\left(1+x^{2}\right)^{1 / 2}} d x .
$$

We now use (A) in Proposition 3

$$
\begin{aligned}
\left|S_{k}\left(u_{n}(1+i x)\right)\right| \leq & S_{k}\left(u_{n} \sqrt{1+x^{2}}\right)= \\
& \sqrt{\frac{2}{\pi}} e^{u_{n} \sqrt{1+x^{2}}} \frac{\zeta u_{n} \sqrt{1+x^{2}}}{u_{n} \sqrt{1+x^{2}}-k} \operatorname{Erfc}(\sqrt{k} \zeta)\left(1+O\left(\frac{1}{\sqrt{k}}\right)\right),
\end{aligned}
$$

where

$$
\zeta=\left|\frac{u_{n} \sqrt{1+x^{2}}}{k}-1-\ln \frac{u_{n} \sqrt{1+x^{2}}}{k}\right|^{1 / 2} .
$$

It is well-known that

$$
\operatorname{Erfc}(\sigma)=\frac{1}{2} e^{-\sigma^{2}} \frac{1}{\sigma}\left(1+O\left(\frac{1}{\sigma^{2}}\right)\right) \quad \text { as } \sigma \rightarrow \infty .
$$

Hence there exists an absolute constant $A$ so that

$$
\operatorname{Erfc}(\sigma) \leq \frac{A}{\sigma+1} e^{-\sigma^{2}} \text { for all } \sigma>0
$$

Putting $\sigma=\sqrt{k} \zeta$ in the above, we have

$$
\operatorname{Erfc}(\sqrt{k} \zeta) \leq A \exp \left(-u_{n} \sqrt{1+x^{2}}+k+k \ln \frac{u_{n} \sqrt{1+x^{2}}}{k}\right) \frac{1}{1+\sqrt{k} \zeta}
$$

uniformly for $k \leq \sqrt{2} u_{n}$ and and $x \geq 1$. 
Combining (3.29), (3.30), and (3.32) and using the estimate $\sqrt{1+x^{2}} \leq 2 x$ for all $x \geq 1$ we have

$$
\begin{aligned}
& \left|\int_{u_{n}}^{\infty} \exp \psi(y) \frac{S_{k}\left(u_{n}+i y\right)-1}{u_{n}+i y} d y\right|= \\
& \quad O\left(e^{k+k \ln \left(u_{n} / k\right)} \int_{1}^{\infty} \exp \left(\left(-\frac{1}{2} e^{u_{n}} u_{n}+k\right) \ln (2 x)\right) d x\right) .
\end{aligned}
$$

It is easily seen that $\int_{1}^{\infty}(2 x)^{-p} d x=O\left(e^{-p / 2}\right)(p>2)$ and $k \ln \left(u_{n} / k\right) \leq u_{n} / e$, for all positive $k$. Therefore

$$
\left|\int_{u_{n}}^{\infty} \exp \psi(y) \frac{S_{k}\left(u_{n}+i y\right)-1}{u_{n}+i y} d y\right|=O\left(e^{-n / 5}\right) .
$$

Returning to (3.26) and making use of the fact [6] that

$$
\frac{n !}{B(n)}=\frac{u_{n}^{n+1} \sqrt{2 \pi\left(e^{u_{n}}+n / u_{n}^{2}\right)}}{\exp \left(e^{u_{n}}-1\right)}\left(1+O\left(\frac{1}{u_{n}}\right)\right),
$$

we have

$$
\begin{aligned}
E\left(\bar{X}_{n, k}\right) & =\frac{u_{n}}{\sqrt{2 \pi}}\left(e^{u_{n}}+n / u_{n}^{2}\right)^{1 / 2}\left(\int_{-\infty}^{\infty} \exp (\psi(y)) \frac{S_{k}\left(u_{n}+i y\right)-1}{u_{n}+i y} d y\right)\left(1+O\left(\frac{1}{u_{n}}\right)\right) \\
& =\frac{u_{n}}{\sqrt{2 \pi}}\left(e^{u_{n}}+n / u_{n}^{2}\right)^{1 / 2}\left(\int_{-\pi}^{\pi} \exp (\psi(y)) \frac{S_{k}\left(u_{n}+i y\right)-1}{u_{n}+i y} d y\right)\left(1+O\left(\frac{1}{u_{n}}\right)\right)
\end{aligned}
$$

uniformly for $k \leq \sqrt{2} u_{n}$.

Now consider the difference

$$
\begin{aligned}
D: & =\int_{-\pi}^{\pi} \exp (\psi(y)) \frac{S_{k}\left(u_{n}+i y\right)-1}{u_{n}+i y} d y-\frac{S_{k}\left(u_{n}\right)}{u_{n}} \int_{-\pi}^{\pi} \exp (\psi(y)) d y \\
& =\int_{-\pi}^{\pi} \exp (\psi(y)) \frac{u_{n}\left(S_{k}\left(u_{n}+i y\right)-S_{k}\left(u_{n}\right)\right)-i y S_{k}\left(u_{n}\right)}{u_{n}\left(u_{n}+i y\right)} d y
\end{aligned}
$$

Take $C_{1}$ to be the contour $\left\{z:\left|z-u_{n}\right|=2 \pi\right\}$ and consider

$$
\begin{aligned}
S_{k}\left(u_{n}+i y\right)-S_{k}\left(u_{n}\right) & =\frac{1}{2 \pi i} \oint_{C_{1}} S_{k}(z)\left(\frac{1}{z-\left(u_{n}+i y\right)}-\frac{1}{z-u_{n}}\right) d z \\
& =\frac{y}{2 \pi} \oint_{C_{1}} S_{k}(z) \frac{d z}{\left(z-u_{n}\right)\left(z-u_{n}-i y\right)}
\end{aligned}
$$

We have

$$
\begin{aligned}
\left|S_{k}\left(u_{n}+i y\right)-S_{k}\left(u_{n}\right)\right| & \leq \frac{|y|}{\pi} \underset{z \in C_{1}}{\operatorname{Max}}\left|S_{k}(z)\right| \\
& =\frac{|y|}{\pi} S_{k}\left(u_{n}+2 \pi\right) .
\end{aligned}
$$


Using (3.39) to estimate the difference $D$ in (3.37), we have

$$
\begin{aligned}
D & \leq \int_{-\pi}^{\pi} \exp (\operatorname{Re}(\psi(y))) \frac{u_{n}\left(|y| S_{k}\left(u_{n}+2 \pi\right) / \pi\right)+|y| S_{k}\left(u_{n}\right)}{u_{n}\left(u_{n}-\pi\right)} d y \\
& \leq \frac{2 S_{k}\left(u_{n}+2 \pi\right)}{\pi\left(u_{n}-\pi\right)} \int_{-\pi}^{\pi} \exp (\operatorname{Re}(\psi(y)))|y| d y \\
& =\frac{4 S_{k}\left(u_{n}+2 \pi\right)}{\pi\left(u_{n}-\pi\right)} \int_{0}^{\pi} \exp (\operatorname{Re}(\psi(y))) y d y .
\end{aligned}
$$

Recall from (3.27) that $\operatorname{Re} \psi(y)=e^{u_{n}}\left(-1+\cos y-u_{n} \ln \left(1+y^{2} u_{n}^{-2}\right)^{1 / 2}\right)$. Hence

$$
\begin{aligned}
\int_{0}^{\pi} \exp (\operatorname{Re}(\psi(y))) y d y & \leq \int_{0}^{\pi} \exp \left(e^{u_{n}}(-1+\cos y)\right) y d y \\
& =\int_{0}^{\pi / 2} \exp \left(e^{u_{n}}(-1+\cos y)\right) y d y+\int_{\pi / 2}^{\pi}(*) d y \\
& =\int_{0}^{\pi / 2} \exp \left(e^{u_{n}}(-1+\cos y)\right) y d y+O\left(\exp \left(-e^{u_{n}}\right)\right) .
\end{aligned}
$$

We write

$$
\begin{aligned}
& \int_{0}^{\pi / 2} \exp \left(e^{u_{n}}(-1+\cos y)\right) y d y \\
& =\int_{0}^{\pi / 2} e^{u_{n}}(-\sin y) \exp \left(e^{u_{n}}(-1+\cos y)\right) \frac{y}{e^{u_{n}}(-\sin y)} d y .
\end{aligned}
$$

Integration by parts provides the estimate

$$
\int_{0}^{\pi / 2} \exp \left(\left(e^{u_{n}}\right)(-1+\cos y)\right) y d y=O\left(e^{-u_{n}}\right) .
$$

Combining (3.41) and (3.42) gives

$$
\int_{0}^{\pi} \exp (\operatorname{Re} \psi(y)) y d y=O\left(e^{-u_{n}}\right) .
$$

By using (3.43), the integral in (3.40) may be estimated

$$
D=O\left(\frac{S_{k}\left(u_{n}+2 \pi\right)}{u_{n}} e^{-u_{n}}\right)
$$

uniformly for $k \geq 1$.

We will require the following three equations:

$$
\begin{gathered}
\int_{-\pi}^{\pi} \exp (\psi(y)) d y=\left(2 \pi e^{-u_{n}}\right)^{1 /, 2}\left(1+O\left(u_{n}^{-1}\right)\right) \\
\int_{-\pi}^{\pi} \exp (\psi(y)) \frac{d y}{u_{n}+i y}=\left(2 \pi e^{-u_{n}}\right)^{1 / 2} u_{n}^{-1}\left(1+O\left(u_{n}^{-1}\right)\right), \\
S_{k}\left(u_{n}+2 \pi\right) \leq S_{k}\left(u_{n}\right) e^{2 \pi}
\end{gathered}
$$


where (3.45) can be found in [6] (see (6.2.4) in [6]), (3.46) can be established the same way as the previous result, and (3.47) can be proved by observing that

$$
\begin{aligned}
\ln S_{k}\left(u_{n}+2 \pi\right)-\ln S_{k}\left(u_{n}\right) & =\int_{u_{n}}^{u_{n}+2 \pi} \frac{S_{k-1}(x)}{S_{k}(x)} d x \\
& \leq \int_{u_{n}}^{u_{n}+2 \pi} 1 d x=2 \pi .
\end{aligned}
$$

Using this gives

$$
D=O\left(\frac{S_{k}\left(u_{n}\right)}{u_{n}} e^{-u_{n}}\right)
$$

uniformly for $k \geq 1$.

Putting (3.45), (3.46), and (3.49) into (3.36), we have

$$
E\left(\bar{X}_{n, k}\right)=S_{k}\left(u_{n}\right)+O\left(\frac{S_{k}\left(u_{n}\right)}{u_{n}}\right)
$$

uniformly for $k \leq \sqrt{2} u_{n}$.

The following proposition deals with the saddle point approximation to the integral $I_{n, k}$ in (3.1). The uniformity in $k$ is the major concern. The usual saddle point method ignores the question of uniformity. Recall equation (3.1) and the definition of the saddle point $\rho_{n, k}$ (see Proposition 1).

\section{Proposition 6.}

$$
I_{n, k}=\frac{\exp \left(e^{\xi / D_{n}}\left(S_{k}\left(\rho_{n, k}\right)-1\right)+R_{k+1}\left(\rho_{n, k}\right)\right)}{\rho_{n, k}^{n+1} \sqrt{2 \pi g_{n, k}^{\prime \prime}\left(\rho_{n, k}\right)}}(1+o(1))
$$

uniformly for $\frac{1}{2} \ln n \leq k \leq \pi n^{5 / 12}$.

Proof. Decompose $I_{n, k}$ as follows:

$$
\begin{aligned}
I_{n, k} & =\frac{1}{2 \pi i} \oint_{|x|=1} \exp \left(g_{n, k}\left(x \rho_{n, k}\right)\right) \frac{d x}{x} \\
& =I_{1}+I_{2},
\end{aligned}
$$

where

$$
I_{1}=\frac{1}{2 \pi i} \oint_{|\theta| \leq \eta} \exp \left(g_{n, k}\left(x \rho_{n, k}\right)\right) \frac{d x}{x}
$$

and

$$
I_{2}=\frac{1}{2 \pi i} \oint_{\eta<|\theta| \leq \pi} \exp \left(g_{n, k}\left(x \rho_{n, k}\right)\right) \frac{d x}{x} .
$$

Here we choose $\eta=n^{-5 / 12}$. We shall show that $I_{1}$ gives the major contribution. First of all

$$
I_{1}=\frac{1}{2 \pi} \int_{-\eta}^{\eta} \exp \left(g_{n, k}\left(e^{i \theta} \rho_{n, k}\right)\right) d \theta
$$


The Taylor expansion of $g_{n, k}\left(z \rho_{n, k}\right)$ at $z=1$ is

$$
g_{n, k}\left(z \rho_{n, k}\right)=g_{n, k}\left(\rho_{n, k}\right)+g_{n, k}^{\prime \prime}\left(\rho_{n, k}\right) \frac{\rho_{n, k}^{2}(z-1)^{2}}{2}+\frac{(z-1)^{3}}{2 \pi i} \oint_{C_{2}} \frac{g_{n, k}\left(\zeta \rho_{n, k}\right)}{(\zeta-z)(\zeta-1)^{3}} d \zeta
$$

where $C_{2}$ is an appropriate contour encircling 1 , and $z$ is in the interior of $C_{2}$. Letting $z=e^{i \theta}$ in the above gives

$$
g_{n, k}\left(e^{i \theta} \rho_{n, k}\right)=g_{n, k}\left(\rho_{n, k}\right)-g_{n, k}^{\prime \prime}\left(\rho_{n, k}\right) \frac{\rho_{n, k}^{2} \theta^{2}}{2}+R(\theta)
$$

where

$$
R(\theta)=\frac{g_{n, k}^{\prime \prime}\left(\rho_{n, k}\right) \rho_{n, k}^{2}}{2}\left(\left(e^{i \theta}-1\right)^{2}+\theta^{2}\right)+\frac{\left(e^{i \theta}-1\right)^{3}}{2 \pi i} \oint_{C_{2}} \frac{g_{n, k}\left(\zeta \rho_{n, k}\right)}{\left(\zeta-e^{i \theta}\right)(\zeta-1)^{3}} d \zeta .
$$

Substituting (3.53) into (3.52), we have

$$
I_{1}=\exp \left(g_{n, k}\left(\rho_{n, k}\right)\right) \frac{1}{2 \pi} \int_{-\eta}^{\eta} \exp \left(-g_{n, k}^{\prime \prime}\left(\rho_{n, k}\right) \rho_{n, k}^{2} \theta^{2} / 2\right) \exp (R(\theta)) d \theta
$$

To estimate $R(\theta)$, we use Proposition 2. Note that $g_{n, k}^{\prime \prime}\left(\rho_{n, k}\right)=e^{\rho_{n, k}}+n / \rho_{n, k}^{2}+$ $\left(e^{\xi / D_{n}}-1\right) S_{k-2}\left(\rho_{n, k}\right)$. Thus

$$
\left|\frac{g_{n, k}^{\prime \prime}\left(\rho_{n, k}\right) \rho_{n, k}^{2}}{2}\left(\left(e^{i \theta}-1\right)^{2}+\theta^{2}\right)\right|=O\left(\theta^{3} n u_{n}\right)=O\left(n^{-1 / 4} u_{n}\right)
$$

uniformly for $k \geq 1$. We now choose the contour $C_{2}=\{\zeta:|\zeta-1|=1 / 12\}$,

$$
\left|\frac{\left(e^{i \theta}-1\right)^{3}}{2 \pi i} \oint_{C_{2}} \frac{g_{n, k}\left(\zeta \rho_{n, k}\right)}{\left(\zeta-e^{i \theta}\right)(\zeta-1)^{3}} d \zeta\right|=O\left(\theta^{3} \oint_{|\zeta-1|=1 / 12} \frac{\left|g_{n, k}\left(\zeta \rho_{n, k}\right)\right|}{\left|\zeta-e^{i \theta}\right||\zeta-1|^{3}}|d \zeta|\right) .
$$

Since $g_{n, k}\left(\zeta \rho_{n, k}\right)=e^{\zeta \rho_{n, k}}-n \ln \left(\zeta \rho_{n, k}\right)+\left(e^{\xi / D_{n}}-1\right) S_{k}\left(\zeta \rho_{n, k}\right)-e^{\xi / D_{n}}$, on $|\zeta-1|=1 / 12$ we have

$$
\begin{aligned}
\left|g_{n, k}\left(\zeta \rho_{n, k}\right)\right| & \leq e^{13 \rho_{n, k} / 12}+O(n \ln \ln n)+O\left(e^{13 \rho_{n, k} / 12} / D_{n}\right) \\
& =O\left(e^{13 u_{n} / 12}\right)=O\left(n^{13 / 12}\right) .
\end{aligned}
$$

Using (3.57), we have from (3.56) that

$$
\left|\frac{\left(e^{i \theta}-1\right)^{3}}{2 \pi i} \oint_{C_{2}} \frac{g_{n, k}\left(\zeta \rho_{n, k}\right)}{\left(\zeta-e^{i \theta}\right)(\zeta-1)^{3}} d \zeta\right|=O\left(n^{-5 / 4} n^{13 / 12}\right)=O\left(n^{-1 / 6}\right)
$$

uniformly for $k \geq 1$.

Combining (3.55) and (3.58) gives

$$
|R(\theta)|=O\left(n^{1 / 6}\right)
$$

uniformly in $k$.

Returning to (3.54), we get

$$
I_{1}=\exp \left(g_{n, k}\left(\rho_{n, k}\right)\right) \frac{1}{2 \pi} \int_{-\eta}^{\eta} \exp \left(-g_{n, k}^{\prime \prime}\left(\rho_{n, k}\right) \rho_{n, k}^{2} \theta^{2} / 2\right) d \theta\left(1+O\left(n^{-1 / 6}\right)\right)
$$


Observe that $g_{n, k}^{\prime \prime}\left(\rho_{n, k}\right) \rho_{n, k}^{2} \sim e^{u_{n}} u_{n}^{2}$ uniformly in $k$ and $\eta=n^{-5 / 12}>n^{-1 / 2}$. By the classical Laplace method, we obtain

$$
\frac{1}{2 \pi} \int_{-\eta}^{\eta} \exp \left(-g_{n, k}^{\prime \prime}\left(\rho_{n, k}\right) \rho_{n, k}^{2} \theta^{2} / 2\right) d \theta=\left(2 \pi g_{n, k}^{\prime \prime}\left(\rho_{n, k}\right) \rho_{n, k}^{2}\right)^{-1 / 2}\left(1+O\left(e^{-n^{1 / 6} / 2}\right)\right) \text {. }
$$

Substituting (3.61) into (3.60) gives

$$
I_{1}=\frac{\exp \left(e^{\xi / D_{n}}\left(S_{k}\left(\rho_{n, k}\right)-1\right)+R_{k+1}\left(\rho_{n, k}\right)\right)}{\rho_{n, k}^{n+1} \sqrt{2 \pi g_{n, k}^{\prime \prime}\left(\rho_{n, k}\right)}}\left(1+O\left(n^{-1 / 6}\right)\right)
$$

uniformly for $k \geq 1$.

Next, we shall show that $I_{2}$ is negligible compared with $I_{1}$. We have

$$
I_{2}=\frac{1}{2 \pi} \int_{\eta<|\theta| \leq \pi} \exp \left(g_{n, k}\left(e^{i \theta} \rho_{n, k}\right)\right) d \theta
$$

It follows that

$$
\begin{aligned}
\left|I_{2}\right| & \leq \frac{1}{2} \exp \left(g_{n, k}\left(\rho_{n, k}\right)\right) \operatorname{Sup}_{\eta<|\theta| \leq \pi}\left(\frac{\left|\exp \left(g_{n, k}\left(e^{i \theta} \rho_{n, k}\right)\right)\right|}{\exp \left(g_{n, k}\left(\rho_{n, k}\right)\right)}\right) \\
& =\frac{1}{2} \exp \left(g_{n, k}\left(\rho_{n, k}\right)\right) \operatorname{Sup}_{\eta<|\theta| \leq \pi} \frac{\exp \left(\operatorname{Re}\left(g_{n, k}\left(e^{i \theta} \rho_{n, k}\right)\right)\right)}{\exp \left(g_{n, k}\left(\rho_{n, k}\right)\right)} .
\end{aligned}
$$

Recall equation (3.1). We have

$$
\begin{aligned}
\operatorname{Sup}_{\eta<|\theta| \leq \pi} \frac{\exp \left(\operatorname{Re}\left(g_{n, k}\left(e^{i \theta} \rho_{n, k}\right)\right)\right)}{\exp \left(g_{n, k}\left(\rho_{n, k}\right)\right)} \\
=\operatorname{Sup}_{\eta<|\theta| \leq \pi} \exp \left\{e^{\xi / D_{n}}\left(\operatorname{Re} S_{k}\left(e^{i \theta} \rho_{n, k}\right)-S_{k}\left(\rho_{n, k}\right)\right)\right. \\
\left.\quad+\left(\operatorname{Re} R_{k+1}\left(e^{i \theta} \rho_{n, k}\right)-R_{k+1}\left(\rho_{n, k}\right)\right)\right\} \\
\leq \operatorname{Sup}_{\eta<|\theta| \leq \pi} \exp \left\{-e^{\xi / D_{n}}\left(S_{k}\left(\rho_{n, k}\right)-\operatorname{Re} S_{k}\left(e^{i \theta} \rho_{n, k}\right)\right)\right\} \\
\leq \exp \left\{-e^{\xi / D_{n}}\left(S_{k}\left(\rho_{n, k}\right)-\operatorname{Re} S_{k}\left(e^{i \eta} \rho_{n, k}\right)\right)\right\} .
\end{aligned}
$$

To proceed further, we need some lower estimates:

$$
S_{k}\left(\rho_{n, k}\right)-\operatorname{Re} S_{k}\left(e^{i \eta} \rho_{n, k}\right)=\sum_{j=0}^{k} \frac{1-\cos \left(j n^{-5 / 12}\right)}{j !} \rho_{n, k}^{j}=2 \sum_{j=0}^{k} \frac{\sin ^{2}\left(j /\left(2 n^{5 / 12}\right)\right)}{j !} \rho_{n, k}^{j} .
$$

Since $j /\left(2 n^{5 / 12}\right) \leq \pi / 2$, by Jordan's inequality ( $\sin x \geq 2 x / \pi$ for $\left.0 \leq x \leq \pi / 2\right)$, we 
have

$$
\begin{aligned}
S_{k}\left(\rho_{n, k}\right)-\operatorname{Re} S_{k}\left(e^{i \eta} \rho_{n, k}\right) & \geq 2\left(\frac{2}{\pi}\right)^{2}\left(\frac{1}{2 n^{5 / 12}}\right)^{2} \sum_{j=0}^{k} \frac{j^{2} \rho_{n, k}^{j}}{j !} \\
& \geq \frac{2}{\pi^{2}} n^{-5 / 6} S_{k}\left(\rho_{n, k}\right) \\
& \geq \frac{2}{\pi^{2}} n^{-5 / 6} S_{k}\left(u_{n}-1\right) \quad \text { (use Proposition 2) } \\
& \geq \frac{2}{\pi^{2}} n^{-5 / 6} S_{k}\left(u_{n}\right) e^{-1} \\
& \geq \frac{2}{e \pi^{2}} n^{-5 / 6} S_{\lfloor(\ln n) / 2\rfloor}\left(u_{n}\right),
\end{aligned}
$$

where $\lfloor(\ln n) / 2\rfloor$ denotes the integer part of $(\ln n) / 2$.

By (A) of Proposition 3, we find

$$
S_{\lfloor(\ln n) / 2\rfloor}\left(u_{n}\right) \geq n^{0.8465} .
$$

Combining (3.65) and (3.66), we get

$$
S_{k}\left(\rho_{n, k}\right)-\operatorname{Re} S_{k}\left(e^{i \eta} \rho_{n, k}\right) \geq \frac{2}{e \pi^{2}} n^{0.8465-5 / 6} \geq \frac{2}{e \pi^{2}} n^{0.0131} .
$$

Putting (3.67) into (3.64) gives

$$
\operatorname{Sup}_{\eta<|\theta| \leq \pi} \frac{\exp \left(\operatorname{Re}\left(g_{n, k}\left(e^{i \theta} \rho_{n, k}\right)\right)\right)}{\exp \left(g_{n, k}\left(\rho_{n, k}\right)\right)} \leq \exp \left(-e^{\xi / D_{n}} \frac{2}{e \pi^{2}} n^{0.0131}\right),
$$

which shows that $I_{2}$ is negligible compared to $I_{1}$.

\section{Determination of the effective block size}

Note that the effective size maintains the expectation of the total number of blocks, i.e., the $M_{n}$ in (1.1) must be at least asymptotic to the $E\left(\bar{X}_{n, k}\right)$ in Proposition 5. According to Proposition 5, the effective size $k$ must be such that $S_{k}\left(u_{n}\right) \sim M_{n}$. By Proposition 4 , the effective size $k$ cannot be less than or equal to $u_{n}$, hence $k>u_{n}$. In summary, we can say that the effective size $k$ must satisfy

$$
\begin{gathered}
k>u_{n}, \\
S_{k}\left(u_{n}\right)=e^{u_{n}}(1+o(1)) .
\end{gathered}
$$

We shall find that the constraint $k \leq \pi n^{5 / 12}$ is sufficient to allow us to analyze the problem. With this constraint on $k$ we are in a position to use Proposition 6. From (2.4) and (3.1), we have

$$
E\left(\exp \left(\xi \frac{\bar{X}_{n, k}-M_{n}}{D_{n}}\right)\right)=e^{-\xi M_{n} / D_{n}} \frac{n !}{B(n)} I_{n, k} .
$$

For clarity, we introduce the notation

$$
\frac{S_{k}\left(\rho_{n, k}\right)}{e^{\rho_{n, k}}}=1+\varepsilon_{n, k}, \quad \frac{S_{k-1}\left(\rho_{n, k}\right)}{e^{\rho_{n, k}}}=1+\tilde{\varepsilon}_{n, k} .
$$

Because of Proposition 2 and (4.2), both $\varepsilon_{n, k}$ and $\tilde{\varepsilon}_{n, k}$ are $o(1)$ uniformly for $k>u_{n}$. 
To simplify (4.3), we use (3.35) and Proposition 6. Rearranging factors in (4.3), we have

$$
\begin{gathered}
E\left(\exp \left(\xi \frac{\bar{X}_{n, k}-M_{n}}{D_{n}}\right)\right)=e^{-\xi M_{n} / D_{n}} \frac{u_{n}}{\rho_{n, k}} \frac{\sqrt{2 \pi\left(e^{u_{n}}+n u_{n}^{-2}\right)}}{\sqrt{2 \pi g_{n, k}^{\prime \prime}\left(\rho_{n, k}\right)}} \exp \left(e^{\rho_{n, k}}-e^{u_{n}}\right)\left(\frac{u_{n}}{\rho_{n, k}}\right)^{n} \\
\times \exp \left(S_{k}\left(\rho_{n, k}\right)\left(e^{\xi / D_{n}}-1\right)\right) \exp \left(1-e^{\xi / D_{n}}\right)(1+o(1)) .
\end{gathered}
$$

Also we have

$$
\begin{gathered}
\frac{u_{n}}{\rho_{n, k}}=1+o(1), \\
\frac{\sqrt{2 \pi\left(e^{u_{n}}+n u_{n}^{-2}\right)}}{\sqrt{2 \pi g_{n, k}^{\prime \prime}\left(\rho_{n, k}\right)}}=1+o(1), \\
\exp \left(1-e^{\xi / D_{n}}\right)=1+o(1) .
\end{gathered}
$$

Putting (4.6), (4.7), and (4.8) into (4.5) gives

$$
\begin{aligned}
& E\left(\exp \left(\xi \frac{\bar{X}_{n, k}-M_{n}}{D_{n}}\right)\right) \\
& \quad=e^{-\xi M_{n} / D_{n}} \exp \left(e^{\rho_{n, k}}-e^{u_{n}}\right)\left(\frac{u_{n}}{\rho_{n, k}}\right)^{n} \exp \left(S_{k}\left(\rho_{n, k}\right)\left(e^{\xi / D_{n}}-1\right)\right)(1+o(1)),
\end{aligned}
$$

uniformly for $\pi n^{5 / 12} \geq k>u_{n}$.

Observe the following:

$$
\begin{gathered}
e^{\rho_{n, k}-e^{u_{n}}}=-e^{u_{n}}\left(1-e^{\rho_{n, k}-u_{n}}\right) \\
=e^{u_{n}}\left(\rho_{n, k}-u_{n}\right)+\frac{1}{2} e^{u_{n}}\left(\rho_{n, k}-u_{n}\right)^{2}+O\left(e^{u_{n}}\left|\rho_{n, k}-u_{n}\right|^{3}\right) . \\
\left(\frac{u_{n}}{\rho_{n, k}}\right)^{n}=\exp \left(-n \ln \frac{\rho_{n, k}}{u_{n}}\right)=\exp \left(-n \ln \left(1+\frac{\rho_{n, k}-u_{n}}{u_{n}}\right)\right) \\
=\exp \left(-\frac{n}{u_{n}}\left(\rho_{n, k}-u_{n}\right)+\frac{n}{2 u_{n}^{2}}\left(\rho_{n, k}-u_{n}\right)^{2}+O\left(\frac{n}{u_{n}^{3}}\left|\rho_{n, k}-u_{n}\right|^{3}\right)\right) .
\end{gathered}
$$

Substituting (4.10) and (4.11) in (4.9), we have

$$
\begin{gathered}
E\left(\exp \left(\xi \frac{\bar{X}_{n, k}-M_{n}}{D_{n}}\right)\right) \\
=e^{-\xi M_{n} / D_{n}} \exp \left(\frac{e^{u_{n}}}{2}\left(\rho_{n, k}-u_{n}\right)^{2}+\frac{n}{2 u_{n}^{2}}\left(\rho_{n, k}-u_{n}\right)^{2}+o(1)\right) \\
\quad \times \exp \left(S_{k}\left(\rho_{n, k}\right)\left(e^{\xi / D_{n}}-1\right)\right)(1+o(1)) .
\end{gathered}
$$


Now using Proposition 2 and (4.4), we have

$$
\begin{aligned}
\frac{e^{u_{n}}}{2}\left(\rho_{n, k}-u_{n}\right)^{2}+\frac{n}{2 u_{n}^{2}} & \left(\rho_{n, k}-u_{n}\right)^{2}+o(1) \\
& =\frac{u_{n}}{2} \xi^{2}\left(1+\tilde{\varepsilon}_{n, k}\right)-\xi^{2}(1+o(1))+\frac{1}{2} \xi^{2}(1+o(1))+o(1) \\
& =\frac{u_{n}}{2} \xi^{2}+\frac{u_{n}}{2} \xi^{2} \tilde{\varepsilon}_{n, k}-\frac{1}{2} \xi^{2}+o(1)
\end{aligned}
$$

Similarly, we have

$$
\begin{aligned}
S_{k}\left(\rho_{n, k}\right)\left(e^{\xi / D_{n}}-1\right) \\
=\left(e^{\rho_{n, k}}+e^{\rho_{n, k}} \varepsilon_{n, k}\right)\left(\frac{\xi}{D_{n}}+\frac{1}{2} \frac{\xi^{2}}{D_{n}^{2}}+O\left(\frac{1}{D_{n}^{3}}\right)\right) \\
=e^{\rho_{n, k}}\left(\frac{\xi}{D_{n}}+\frac{1}{2} \frac{\xi^{2}}{D_{n}^{2}}+O\left(\frac{1}{D_{n}^{3}}\right)\right)+e^{\rho_{n, k}} \varepsilon_{n, k}\left(\frac{\xi}{D_{n}}+O\left(\frac{1}{D_{n}^{2}}\right)\right) \\
=\frac{e^{u_{n}}}{D_{n}} \xi-u_{n} \xi^{2}\left(1+\tilde{\varepsilon}_{n, k}\right)+\frac{1}{2} u_{n} \xi^{2}+\xi^{2}+o(1)+e^{\rho_{n, k}} \varepsilon_{n, k}\left(\frac{\xi}{D_{n}}+O\left(\frac{1}{D_{n}^{2}}\right)\right) .
\end{aligned}
$$

These expressions hold uniformly for $\pi n^{5 / 12} \geq k>u_{n}$.

Putting (4.13) and (4.14) in (4.12) gives

$$
\begin{aligned}
& E\left(\exp \left(\xi \frac{\bar{X}_{n, k}-M_{n}}{D_{n}}\right)\right) \\
& \quad=\exp \left(-\frac{1}{2} u_{n} \xi^{2} \tilde{\varepsilon}_{n, k}+\frac{1}{2} \xi^{2}+e^{\rho_{n, k}} \varepsilon_{n, k}\left(\frac{\xi}{D_{n}}+O\left(\frac{1}{D_{n}^{2}}\right)\right)\right)(1+o(1)) .
\end{aligned}
$$

We summarize our findings in the following proposition.

Proposition 7. The Laplace transform of the random variable $\left(\bar{X}_{n, k}-M_{n}\right) / D_{n}$, i.e., $E\left(\exp \left(\xi\left(\bar{X}_{n, k}-M_{n}\right) / D_{n}\right)\right)$ is equal to

$$
\exp \left(-\frac{1}{2} u_{n} \xi^{2} \tilde{\varepsilon}_{n, k}+\frac{1}{2} \xi^{2}+e^{\rho_{n, k}} \varepsilon_{n, k}\left(\frac{\xi}{D_{n}}+O\left(\frac{1}{D_{n}^{2}}\right)\right)\right)(1+o(1))
$$

uniformly for $\pi n^{5 / 12} \geq k>u_{n}$, where $\tilde{\varepsilon}_{n, k}$ and $\varepsilon_{n, k}$ are defined in (4.4).

The presence of the term $\frac{1}{2} \xi^{2}$ is an indication of the asymptotic normality of the distribution. It is clear that the effective size $k$ must make

$$
-\frac{1}{2} u_{n} \xi^{2} \tilde{\varepsilon}_{n, k}+e^{\rho_{n . k}} \varepsilon_{n, k}\left(\frac{\xi}{D_{n}}+O\left(\frac{1}{D_{n}^{2}}\right)\right)=o(1)
$$

Proposition 8. If $\pi n^{5 / 12} \geq k>u_{n}$ and $\liminf \left(u_{n} / k\right) \geq 3 / 4$, then $E\left(\exp \left(\xi\left(\bar{X}_{n, k}-\right.\right.\right.$ $\left.\left.M_{n}\right) / D_{n}\right)$ ) does not converge to $e^{\xi^{2} / 2}$. 
Proof. We know already that $S_{k}\left(\rho_{n, k}\right) / e^{\rho_{n, k}}=1+\varepsilon_{n, k}$, where $\varepsilon_{n, k}=o(1)$. But this is not strong enough to force the conclusion. We need more detailed information about $\varepsilon_{n, k}$. Using Proposition 2 gives

$$
\frac{S_{k}\left(\rho_{n, k}\right)}{e^{\rho_{n . k}}}=\frac{S_{k}\left(u_{n}+O\left(1 / D_{n}\right)\right)}{e^{u_{n}+O\left(1 / D_{n}\right)}} .
$$

By an argument similar to (3.48), one can show that

$$
S_{k}\left(u_{n}+O\left(\frac{1}{D_{n}}\right)\right)=S_{k}\left(u_{n}\right) e^{O\left(\frac{1}{D_{n}}\right)} .
$$

Hence

$$
\frac{S_{k}\left(\rho_{n, k}\right)}{e^{\rho_{n . k}}}=\frac{S_{k}\left(u_{n}\right)}{e^{u_{n}}}\left(1+O\left(1 / D_{n}\right)\right)=\frac{S_{k}\left(u_{n}\right)}{e^{u_{n}}}+O\left(1 / D_{n}\right) .
$$

By (A) of Proposition 3 we find that

$$
\frac{S_{k}\left(u_{n}\right)}{e^{u_{n}}}=1+\sqrt{\frac{2}{\pi}} \frac{\zeta x}{x-1} \operatorname{Erfc}(\sqrt{k} \zeta)\left(1+O\left(\frac{1}{\sqrt{k}}\right)\right),
$$

where $x=u_{n} / k<1$ and $\zeta=|x-1-\ln x|^{1 / 2}$.

Plugging (4.17) into (4.16) gives

$$
\varepsilon_{n, k}=\sqrt{\frac{2}{\pi}} \frac{\zeta x}{x-1} \operatorname{Erfc}(\sqrt{k} \zeta)\left(1+O\left(\frac{1}{\sqrt{k}}\right)\right)+O\left(1 / D_{n}\right) .
$$

Observe that $\varepsilon_{n, k}=o(1), O\left(1 / D_{n}\right)=o(1)$ and that $\zeta /(x-1)$ is bounded. Hence

$$
\operatorname{Erfc}(\sqrt{k} \zeta)=o(1)
$$

This implies

$$
\sqrt{k} \zeta \rightarrow \infty \quad \text { as } k \rightarrow \infty .
$$

Once we are guaranteed that the argument $\sigma$ of $\operatorname{Erfc}(\sigma)$ tends to $\infty$, we can use the traditional asymptotics,

$$
\operatorname{Erfc}(\sigma)=\frac{1}{2 \sigma} e^{-\sigma^{2}}\left(1+O\left(1 / \sigma^{2}\right)\right) \quad \text { as } \sigma \rightarrow \infty .
$$

Thus

$$
\begin{aligned}
\operatorname{Erfc}(\sqrt{k} \zeta) & =\frac{1}{2 \sqrt{k} \zeta} e^{-k \zeta^{2}}\left(1+O\left(\frac{1}{k \zeta^{2}}\right)\right) \\
& \geq \frac{1}{3} \exp \left(-k\left(\frac{u_{n}}{k}-1-\ln \frac{u_{n}}{k}\right)\right)
\end{aligned}
$$

for $n$ large.

Substituting (4.18) and (4.22) into the term $e^{\rho_{n, k}} \varepsilon_{n, k} / D_{n}$ gives

$$
\left|\frac{e^{\rho_{n, k}} \varepsilon_{n, k}}{D_{n}}\right| \geq \frac{1}{3} \sqrt{\frac{2}{\pi}}\left|\frac{\zeta x}{x-1}\right| \frac{e^{\rho_{n . k}}}{D_{n}} \exp \left(-k\left(\frac{u_{n}}{k}-1-\ln \frac{u_{n}}{k}\right)\right)-l_{n},
$$

where $\left|l_{n}\right|=O\left(e^{\rho_{n . k}} / D_{n}^{2}\right)=O(\ln n)$. 
Note that $|\zeta x /(x-1)|$ is non-zero and bounded. Using Proposition 2, we have

$$
\begin{aligned}
\frac{e^{\rho_{n, k}}}{D_{n}} \exp \left(-k\left(\frac{u_{n}}{k}\right.\right. & \left.\left.-1-\ln \frac{u_{n}}{k}\right)\right) \\
& =\exp \left(k\left(1+\ln \frac{u_{n}}{k}\right)-\frac{\ln n}{2}+\ln u_{n}+O\left(1 / D_{n}\right)\right) \\
& \geq \exp \left(u_{n}(1+\ln 5 / 8)-\frac{\ln n}{2}+\ln u_{n}\right) \quad\left(\text { use lim inf } u_{n} / k \geq 3 / 4\right) \\
& \geq \exp \left(u_{n}(0.5299-0.5)+\ln u_{n}\right) \\
& =\exp \left(u_{n}(0.0299)+\ln u_{n}\right) .
\end{aligned}
$$

From (4.23) and (4.24), it is clear that $\left|e^{\rho_{n . k}} \varepsilon_{n, k} / D_{n}\right| \geq e^{0.0298 u_{n}}$; the asymptotic magnitude is much larger than that of $-u_{n} / 2 \xi^{2} \tilde{\varepsilon}_{n, k}$ which is $O(\ln n)$. Hence

$$
-\frac{1}{2} u_{n} \xi^{2} \tilde{\varepsilon}_{n, k}+e^{\rho_{n, k}} \varepsilon_{n, k}\left(\frac{\xi}{D_{n}}+O\left(\frac{1}{D_{n}^{2}}\right)\right)
$$

is not $o(1)$. By Proposition 7, Proposition 8 is proved.

According to Proposition 8 , the possible effective size $k$ must be such that $u_{n} / k \leq$ 3/4. We shall use Proposition 3 to find it. We have

$$
\frac{S_{k}(k x)}{e^{k x}}=1-\frac{1}{\sqrt{2 k \pi}} \frac{x}{1-x}\left(x e^{1-x}\right)^{k}\left(1+O\left(\frac{1}{k}\right)\right)
$$

uniformly for $0<x \leq 3 / 4$.

Let $x=\rho_{n, k} / k$ in (4.25). We have

$$
S_{k}\left(\rho_{n, k}\right)=e^{\rho_{n, k}}-\frac{e^{\rho_{n, k}}}{\sqrt{2 k \pi}} \frac{x}{1-x}\left(x e^{1-x}\right)^{k}\left(1+O\left(\frac{1}{k}\right)\right) .
$$

Comparing (4.26) with (4.4) shows

$$
\begin{aligned}
e^{\rho_{n, k}} \varepsilon_{n, k} & =-\frac{e^{\rho_{n, k}}}{\sqrt{2 k \pi}} \frac{x}{1-x}\left(x e^{1-x}\right)^{k}\left(1+O\left(\frac{1}{k}\right)\right) \\
& =-\frac{1}{\sqrt{2 \pi}} \frac{x}{1-x} \exp \left(\rho_{n, k}+k \ln \left(x e^{1-x}\right)-\frac{\ln k}{2}\right)(1+O(1 / k))
\end{aligned}
$$

Thus

$$
\begin{aligned}
\frac{e^{\rho_{n . k}} \varepsilon_{n, k}}{D_{n}}=- & \frac{1}{\sqrt{2 \pi}} \frac{x}{1-x} \exp \left(\rho_{n, k}+k \ln \left(x e^{1-x}\right)-\frac{\ln k}{2}-\frac{\ln n}{2}+\ln u_{n}\right) \\
& \times(1+O(1 / k)) .
\end{aligned}
$$

We must force $e^{\rho_{n, k}} \varepsilon_{n, k} / D_{n}$ to be $o(1)$. Because, in general, $e^{\rho_{n, k}} \varepsilon_{n, k} / D_{n} \geq u_{n} \tilde{\varepsilon}_{n, k}$, when this is done the term $-\frac{1}{2} u_{n} \xi^{2} \tilde{\varepsilon}_{n, k}$ also simultaneously becomes $o(1)$. Then, by Proposition 7 , the asymptotic normality of the random variable $\left(\bar{X}_{n, k}-M_{n}\right) / D_{n}$ will follow. We now use Proposition 2 to simplify (4:28),

$$
\begin{aligned}
\frac{e^{\rho_{n, k}} \varepsilon_{n, k}}{D_{n}}=- & \frac{1}{\sqrt{2 \pi}} \frac{x}{1-x} \exp \left(-k \ln k+k \ln u_{n}+k-\frac{\ln k}{2}-\frac{\ln n}{2}+\ln u_{n}+o(1)\right) \\
& \times(1+O(1 / k)) .
\end{aligned}
$$


The optimal choice for $k$ must satisfy

$$
-k \ln k+k \ln u_{n}+k-\frac{\ln k}{2}-\frac{\ln n}{2}+\ln u_{n}=0 .
$$

We emphasize that (4.30) is the equation for the effective size $k$. It is obvious from the expression (4.30) that if we replace $k$ by $k+L_{n}$ where $L_{n} \rightarrow \infty$ as $n \rightarrow \infty$, then $e^{\rho_{n . k}} \varepsilon_{n, k} / D_{n}$ is $o(1)$. Consequently the random variable $\left(\bar{X}_{n, k}-M_{n}\right) / D_{n}$ is asymptotically normal.

\section{Asymptotics for the effective size and the transitional distributions}

To study equation (4.30), we first must investigate the positive roots of the equation

$$
f_{n}(z)=\frac{1}{2} \ln n-\ln u_{n}
$$

where $f_{n}(z):=-z \ln z+z \ln u_{n}+z-\frac{1}{2} \ln z$.

Proposition 9. For all sufficiently large $n, f_{n}(z)=\frac{1}{2} \ln n-\ln u_{n}$ has three positive roots. The relative maximum of $f_{n}(z)$ occurs approximately at $u_{n}$.

The proof uses only elementary calculus, and we omit it.

Denote by $\mu_{n}$ the largest positive root of (5.1). Hence $\mu_{n}>u_{n}$ so $\mu_{n}$ is the effective block size of the problem. In order to study the asymptotics of $\mu_{n}$, we make a change of variable, $y_{n}=u_{n} / \mu_{n}$. Plugging this into (5.1) we have

$$
u_{n} \ln y_{n}+u_{n}-\frac{1}{2} y_{n} \ln \frac{u_{n}}{y_{n}}=y_{n}\left(\frac{1}{2} \ln n-\ln u_{n}\right) \text {. }
$$

Let $h_{n}(y)=u_{n} \ln y+u_{n}-\frac{1}{2} y \ln \left(u_{n} / y\right)-y\left(\frac{1}{2} \ln n-\ln u_{n}\right)$. By Proposition 9 the function $h_{n}(y)$ has a unique positive root $y_{n}$ in the interval $(0,1)$ provided that $n$ is sufficiently large.

Proposition 10. $y_{n}$ admits the asymptotic approximation

$$
y_{n}=r+\frac{r^{2} \ln (1 / r)}{2-r} \frac{1}{u_{n}}+o\left(\frac{1}{u_{n}}\right),
$$

where $r$ is the unique positive root of $\frac{1}{2} z-\ln z-1=0$ in $(0,1)$.

Proof. The main issue here is to show $y_{n}$ has a limit. Its asymptotics are obtained by the same bootstrap method as used previously,

$$
h_{n}(1 / 2)=u_{n}(1-\ln 2)-\frac{1}{4} \ln \left(2 u_{n}\right)-\frac{1}{4} \ln n+\frac{1}{2} \ln u_{n} .
$$

Since $1-\ln 2>1 / 4, h_{n}(1 / 2)>0$ if $n$ is large. Similarly, $h_{n}(1 / 3)<0$ if $n$ is large. Hence

$$
\frac{1}{3}<y_{n}<\frac{1}{2} \quad \text { for all large } n .
$$

Let $\bar{r}:=\lim \sup y_{n}$. We have

$$
\frac{1}{3} \leq \bar{r} \leq \frac{1}{2}
$$


Because the logarithm is a continuous function on $(0,1)$, we have

$$
\lim \sup \ln y_{n}=\ln \bar{r} \text {. }
$$

Now consider the equation satisfied by $y_{n}$ :

$$
\ln y_{n}+1=\frac{1}{2} \frac{y_{n}}{u_{n}} \ln \frac{u_{n}}{y_{n}}+y_{n}\left(\frac{1}{2} \frac{\ln n}{u_{n}}-\frac{\ln u_{n}}{u_{n}}\right) .
$$

Taking lim sup in (5.7), we get

$$
\lim \sup \left(\ln y_{n}+1\right)=\lim \sup \left(\frac{1}{2} \frac{y_{n}}{u_{n}} \ln \frac{u_{n}}{y_{n}}+y_{n}\left(\frac{1}{2} \frac{\ln n}{u_{n}}-\frac{\ln u_{n}}{u_{n}}\right)\right) .
$$

To simplify (5.8), we observe that $\lim \sup \left(a_{n}+b_{n}\right)=\lim \sup a_{n}+\lim \sup b_{n}$ if $b_{n}$ is a null sequence or a constant sequence. Notice that

$$
\frac{1}{2} \frac{y_{n}}{u_{n}} \ln \frac{u_{n}}{y_{n}}=o(1) \quad \text { and } \quad \lim \left(\frac{1}{2} \frac{\ln n}{u_{n}}-\frac{\ln u_{n}}{u_{n}}\right)=1 / 2 .
$$

We have

$$
\ln \bar{r}+1=\frac{1}{2} \bar{r} .
$$

That is, $\bar{r}$ satisfies $z / 2-\ln z-1=0$. Similarly, one shows that $\lim \inf y_{n}$ also satisfies $z / 2-\ln z-1=0$. Since $z / 2-\ln z-1=0$ has a unique root $r$ in $(0,1)$, we find that $\lim y_{n}=r$.

Now we bootstrap. Let $y_{n}=r+\varepsilon_{n}$ with $\varepsilon_{n}=o(1)$ and plug this into (5.7),

$$
\ln \left(r+\varepsilon_{n}\right)+1-\frac{1}{2} \frac{r+\varepsilon_{n}}{u_{n}} \ln \frac{u_{n}}{r+\varepsilon_{n}}-\left(r+\varepsilon_{n}\right)\left(\frac{1}{2} \frac{\ln n}{u_{n}}-\frac{\ln u_{n}}{u_{n}}\right)=0 .
$$

To simplify (5.10), we use the following:

$$
\begin{gathered}
\frac{\ln n}{2 u_{n}}-\frac{\ln u_{n}}{u_{n}}=\frac{1}{2}-\frac{\ln u_{n}}{2 u_{n}}, \\
-\frac{r+\varepsilon_{n}}{2 u_{n}} \ln \frac{u_{n}}{r+\varepsilon_{n}}=-\frac{r}{2 u_{n}} \ln \frac{u_{n}}{r}+\frac{r}{2 u_{n}} \ln \left(1+\frac{\varepsilon_{n}}{r}\right) \\
-\frac{\varepsilon_{n}}{2 u_{n}} \ln \frac{u_{n}}{r}+\frac{\varepsilon_{n}}{2 u_{n}} \ln \left(1+\frac{\varepsilon_{n}}{r}\right) .
\end{gathered}
$$

We plug (5.11) and (5.12) in (5.10) to get

$$
\varepsilon_{n}\left(\frac{1}{r}-\frac{1}{2}\right)-\frac{r}{2 u_{n}} \ln \frac{1}{r}+O\left(\varepsilon_{n}^{2}\right)+O\left(\varepsilon_{n} \frac{\ln u_{n}}{u_{n}}\right)=0
$$

or

$$
\varepsilon_{n} \sim\left(\frac{1}{r}-\frac{1}{2}\right)^{-1} \frac{r \ln (1 / r)}{2 u_{n}} \quad \text { as } n \rightarrow \infty
$$

That is

$$
\varepsilon_{n}=\frac{r^{2} \ln (1 / r)}{2-r} \frac{1}{u_{n}}+o\left(\frac{1}{u_{n}}\right)
$$


Theorem 2. The effective size

$$
\mu_{n}=\frac{u_{n}}{r}-\frac{\ln (1 / r)}{2-r}+o(1)
$$

Proof. Use Proposition 10 and the fact that $\mu_{n}=u_{n} / y_{n}$.

The effective size $\mu_{n}$ has the property that it is optimal and guarantees the random variable $\left(\bar{X}_{n, \mu_{n}+L_{n}}-M_{n}\right) / D_{n}$ is asymptotically normal for all $L_{n}$ satisfying $L_{n} \rightarrow$ $\infty$ as $n \rightarrow \infty$. If $L_{n}$ is a bounded sequence, then the corresponding behavior of $\left(\bar{X}_{n, \mu_{n}+L_{n}}-M_{n}\right) / D_{n}$ is called the transitional behavior. Let

$$
k=\left\lfloor\mu_{n}\right\rfloor+L_{n},
$$

where $\left|L_{n}\right| \leq M$ is a bounded integer sequence.

To study the transitional behavior, we return to (4.29)

$$
\begin{aligned}
\frac{e^{\rho_{n, k}} \varepsilon_{n, k} \xi}{D_{n}}=- & \frac{\xi}{\sqrt{2 \pi}} \frac{x}{1-x} \exp \left(-k \ln k+k \ln u_{n}+k-\frac{\ln k}{2}-\frac{\ln n}{2}+\ln u_{n}+o(1)\right) \\
& \times(1+O(1 / k))
\end{aligned}
$$

where $x=\rho_{n, k} / k$. Using (5.15) to simplify (5.16) gives

$$
-k \ln k+k \ln u_{n}+k-\frac{\ln k}{2}-\frac{\ln n}{2}+\ln u_{n}=\left(L_{n}-\left\{\mu_{n}\right\}\right) \ln r+o(1),
$$

where $\left\{\mu_{n}\right\}$ denotes the fractional part of $\mu_{n}$. Furthermore

$$
\frac{x}{1-x}=\frac{r}{1-r}+o(1) \text {. }
$$

Substituting (5.17) and (5.18) into (5.16) gives

$$
\frac{e^{\rho_{n, k}} \varepsilon_{n, k} \xi}{D_{n}}=-\frac{\xi}{\sqrt{2 \pi}} \frac{r}{1-r} r^{L_{n}-\left\{\mu_{n}\right\}}(1+o(1)) .
$$

Put (5.19) in (4.15) of Proposition 7 and observe that $-\frac{1}{2} u_{n} \xi^{2} \tilde{\varepsilon}_{n, k}=o(1)$. Thus

$$
E\left(\exp \left(\xi \frac{\bar{X}_{n, k}-M_{n}}{D_{n}}\right)\right)=\exp \left(-\frac{\xi}{\sqrt{2 \pi}} \frac{r}{1-r} r^{L_{n}-\left\{\mu_{n}\right\}}\right) \exp \left(\frac{1}{2} \xi^{2}+o(1)\right)(1+o(1))
$$

This implies that

$$
E\left(\exp \left(\xi \frac{\bar{X}_{n, k}-\bar{M}_{n}}{D_{n}}\right)\right)=\exp \left(\frac{1}{2} \xi^{2}\right)+o(1)
$$

where

$$
\bar{M}_{n}=M_{n}-\frac{r^{L_{n}+1-\left\{\mu_{n}\right\}}}{\sqrt{2 \pi}(1-r)} D_{n}
$$

Thus we have proved the following:

Theorem 3 (The transitional distribution). If $k=\left\lfloor\mu_{n}\right\rfloor+L_{n}$ where $L_{n}$ is a bounded integer sequence, then the random variable $\left(\bar{X}_{n, k}-\bar{M}_{n}\right) / D_{n}$ is still asymptotically normal, where $\bar{M}_{n}$ is defined in (5.20). 
Remark. The transitional distribution is still normal, but the mean $\bar{M}_{n}$ is different from $M_{n}$. More interestingly, the mean $\bar{M}_{n}$ is an oscillatory function of $n$ due to the following.

Proposition 11. The sequence $\left\{\mu_{n}\right\}$ is dense in $[0,1]$.

Proof. Let $t$ be a large positive number, and let $u_{t}$ be the unique positive root of $z e^{z}=t$. Thus when $t$ is an integer $n, u_{t}$ is reduced to $u_{n}$. Consider the equation in $z$

$$
-z \ln z+z \ln u_{t}+z-\frac{1}{2} \ln z=\frac{1}{2} \ln t-\ln u_{t} .
$$

Let $\mu(t)$ be the largest positive root of (5.21). Thus when $t$ is an integer $n, \mu(t)$ is reduced to $\mu_{n}$. To proceed we mention the following simple facts:

(a) $\mu(t)$ is well-defined for all sufficiently large $t$.

(b) $\mu(t)$ is a differentiable function of $t$.

(c) Since $u_{t}$ satisfies $z e^{z}=t$, we have

$$
u_{t}^{\prime}=\left(e^{u_{t}}+t\right)^{-1} .
$$

To show $\mu(t)$ is a strictly increasing function, we differentiate the equation below with respect to $t$ :

$$
-\mu(t) \ln \mu(t)+\mu(t) \ln u_{t}+\mu(t)-\frac{1}{2} \ln \mu(t)=\frac{1}{2} \ln t-\ln u_{t} .
$$

Thus

$$
\mu^{\prime}(t)\left(-\ln \mu(t)+\ln u_{t}-\frac{1}{2 \mu(t)}\right)=\frac{1}{2 t}-\frac{1+\mu(t)}{t+t u_{t}} .
$$

Since $\mu(t)>u_{t}$, we have

$$
-\ln \mu(t)+\ln u_{t}-\frac{1}{2 \mu(t)}<0 .
$$

By Theorem 1 (when $n$ is replaced by $t$ the same conclusion still holds) we see

$$
\frac{1+\mu(t)}{t+t u_{t}} \sim \frac{1}{t r}>\frac{1}{t}
$$

This implies that

$$
\frac{1}{2 t}-\frac{1+\mu(t)}{t+t u_{t}}<0
$$

Hence $\mu^{\prime}(t)>0$ for all sufficiently large $t$ so $\mu(t)$ is a continuous, strictly increasing function of $t$ that tends to infinity as $t \rightarrow \infty$. Let $K(t)$ be its inverse so that

$$
\mu(K(t))=K(\mu(t))=t .
$$

Applying Theorem 1 to (5.23), we get

$$
\mu^{\prime}(t) \rightarrow 0^{+} \text {as } t \rightarrow \infty \text {. }
$$

Hence

$$
K^{\prime}(t)=\frac{1}{\mu^{\prime}(K(t))} \rightarrow \infty \quad \text { as } \quad t \rightarrow \infty
$$


Now given any $a$ and $b$ such that $0<a<b<1$, by the mean value theorem we have

$$
K(b+l)-K(a+l)=K^{\prime}(\xi)(b-a) \text { for some } \xi \text { between } a+l \text { and } b+l .
$$

By $(5.28) K^{\prime}(\xi) \rightarrow \infty$ as $l \rightarrow \infty$. Hence there exist integers $n_{0}$ and $l_{0}$ such that

$$
K\left(b+l_{0}\right)>n_{0}>K\left(a+l_{0}\right) .
$$

This implies $\mu\left(K\left(b+l_{0}\right)\right)>\mu\left(n_{0}\right)>\mu\left(K\left(a+l_{0}\right)\right)$, and $b+l_{0}>\mu_{n_{0}}>a+l_{0}$. Consequently $\left\{\mu_{n_{0}}\right\} \in(a, b)$.

\section{References}

1. M. Abramowitz and I. A. Stegun, eds., Handbook of Mathematical Functions, New York, 1965.

2. E. A. Bender, Central and local limit theorems applied to asymptotic enumeration, J. Combin. Theory Ser. A 15 (1973), 91-111.

3. J. Curtiss, A note on the theory of moment generating functions, Ann. Math. Statist. 13 (1942), 430-433.

4. L. Harper, Stirling behavior is asymptotically normal, Ann. Math. Statist. 38 (1967), 410-414.

5. M. Kac, Probability methods in some problems of analysis and number theory, Bull. Amer. Math. Soc. 55 (1949), 641-665.

6. N. G. de Bruijn, Asymptotic Methods in Analysis, Dover, New York, 1981.

7. V. N. Sachkov, Random partitions of sets, Theory Probab. Appl. 190 (1974), 184-190.

8. G. Szegö, Uber eine Eigenschaft der Exponentialreihe, Sitzungsber. Berl. Math. Ges. 23 (1924), 50-64.

9. N. M. Temme, The asymptotic expansion of the incomplete gamma functions, SIAM J. Math. Anal. 10 (1979), 757-766.

10. R. S. Varga, Topics in Rational Interpolation and Approximation, Les Presses d l'Université de Montreal, Montreal, Canada, 1982.

11. H. Wilf, generatingfunctionology, Academic Press, 1990.

Department of Mathematics and Computer Science, Drexel University, Philadelphia, PennSYLVANIA 19104 , U. S. A. 